\title{
Control de Phytophthora capsici Leonian en Capsicum annuum cv. Papri king con fungicidas, fertilizantes y biocontroladores
}

\author{
Control of Phytophthora capsici Leonian in Capsicum annuum cv. king papri \\ with fungicides, fertilizers and biocontrol agents
}

Carlos A. Huallanca V. ${ }^{1}$; Carlos A. Cadenas G. ${ }^{2}$

\begin{abstract}
Resumen
Se evaluó la efectividad de tres productos químicos: metalaxil + mancozeb, fosfonato de potasio y sulfato de cobre pentahidratado y de tres biocontroladores: Trichoderma viride, T. harzianum y Bacillus subtilis, en la inhibición del crecimiento de colonias de Phytophthora capsici aisladas inicialmente de cuello y raíces de pimiento 'Papri King' con síntomas de pudriciones. También se evaluó la efectividad de los mismos productos químicos y biocontroladores en la prevención de las pudriciones de cuello y raíces de pimiento 'Papri King'. Se obtuvo que el fungicida con Metalaxyl + Mancozeb inhibió al $100 \%$ el crecimiento de las colonias de Phytophtora capsici, mientras que el Fosfonato de potasio y el Sulfato de cobre no resultaron ser efectivos para inhibir totalmente el crecimiento de $P$. capsici. Los biocontroladores T. viride, T. harzianum y B. sultilis causaron un menor desarrollo de las colonias de $P$. capsici y, adicionalmente, los Trichodermas actuaron parasitando las colonias de P. capsici. El fungicida Metalaxyl + Mancozeb previno totalmente la aparición de pudriciones de cuello y raíces en pimientos causada por $P$. capsici, mientras que el Fosfonato de potasio, el Sulfato de cobre, T. viride, T. harzianum y B. sultilis, no fueron efectivos para prevenir la pudrición de las raíces causada por $P$. capsici en pimientos.
\end{abstract}

Palabras Clave: Phytophthora capsici; Capsicum annuum.

\begin{abstract}
Effectiveness was evaluated of three chemicals: metalaxyl + mancozeb, potassium phosphonate and pentahydrate copper sulfate, and three biocontrol agents: Trichoderma viride, T. harzianum, and Bacillus subtilis in the inhibition of growth of colonies of Phytophthora capsici initially isolated from neck and roots of pepper 'Papri King' with symptoms of rot. We also evaluated the effectiveness of the same chemical products and biocontrol agents in the prevention of neck and root rot of pepper 'Papri King'. It was found that the fungicide Mancozeb + Metalaxyl, inhibited $100 \%$ of the growth of colonies of Phytophthora capsici whereas the potassium phosphonate and copper sulfate were not completely effective in inhibiting the growth of $P$. capsici. The biocontrol agents $T$. viride, T. harzianum and B. subtilis, caused less development of the colonies of $P$. capsici by antibiosis, and additionally trichodermas parasited colonies of $P$. capsici. The fungicide Metalaxyl + Mancozeb completely prevented the occurrence of neck and root rot in pepper caused by $P$. capsici in the greenhouse, while potassium phosphonate, copper sulfate, T. viride, T. harzianum and B. subtilis, were not effective to prevent root rot caused by $P$. capsici on peppers in greenhouse conditions.
\end{abstract}

Keywords: Phytophthora capsici; Capsicum annuum.

\section{Introducción}

El ají páprika es uno de los principales productos que se cultivan en el Perú y está destinado especialmente a la exportación, convirtiéndose en uno de los principales productos de esta actividad. Las principales zonas de producción de ají paprika en el Perú son Tacna, Majes, Ica, Chincha, Cañete, Huaral, Barranca, Casma, Virú, Paiján, Motupe y Piura (Casas, 1994, Nicho, 2004; Lara, 2006).
Los diferentes reportes indican que el principal patógeno que afecta el cultivo del ají páprika es la Phytophthora capsici, produciendo la pudrición radicular y del cuello de la planta, causando susceptibilidad a la marchitez vascular y pudiendo ocasionar la muerte de toda una plantación, lo que ocasiona dificultades en su comercialización y, sobre todo, grandes pérdidas económicas a los productores (Farr y Bills, 1989; Ocampo, 2003).

1. Especialidad de Fitopatología de la Escuela de Post Grado. Universidad Nacional Agraria La Molina, Lima (Perú). Email: chuallanca@gmail.com 2. Departamento de Fitopatología. Universidad Nacional Agraria La Molina, Lima (Perú). Email: ccadenas@lamolina.edu.pe 
La pudrición radicular del pimiento causada por $P$. capsici es una de las enfermedades de mayor importancia económica del género Capsicum alrededor del mundo. Se presentan en cualquier ambiente donde las temperaturas oscilan entre los 15 y $33{ }^{\circ} \mathrm{C}$ y donde el suelo es lo suficientemente húmedo. Este patógeno afecta el sistema radicular destruyendo plántulas y plantas de cualquier edad. $P$. capsici requiere una temperatura mínima de 10 ${ }^{\circ} \mathrm{C}$, pero su temperatura óptima es de $28^{\circ} \mathrm{C}$, no se producen zoosporangios a $18{ }^{\circ} \mathrm{C}$ o a temperaturas más bajas, ni a temperaturas de $35^{\circ} \mathrm{C}$ o superiores a esta (Agrios, 1998; Erwin and Ribeiro, 1996; Lamour y Hausbeck, 2002).

En el control de la P. capsici, como medidas preventivas, se recomienda el uso de suelos planos, livianos, con buen drenaje y libre de malezas y efectuar siembras normales o bien ralas, nunca densas. Se recomienda también la rotación de cultivos usando especies que no sean cucurbitáceas, ni tomate ni berenjena, así como tratamientos con fungicidas de acción sistémica como medida preventiva y curativa (Valiela, 1978; Farr \& Bills, 1989; Mont, 1993; Erwin \& Ribeiro, 1996).

En un estudio de la capacidad antagónica de la bacteria Bacillus subtilis, para controlar enfermedades causadas por $P$. capsici en plantas de tomate y pimiento, se redujo la podredumbre causada por $P$. capsici en un $37 \%$ en condiciones de campo y en un 55,6\% en pimientos cultivados en condiciones de invernaderos. Se ha asegurado la inocuidad de la bacteria al adicionarse a semillas y plántulas de pimiento (Mohammed et al., 2004).

Diversas pruebas realizadas en Capsicum, donde se trató con el hongo bicontrolador Trichoderma harzianum, demuestran que en aquellas plantas a las que se aplicó $T$. harzianum, hubo ausencia del patógeno, posiblemente por una competición de nutrientes, mientras que en aquellas plantas donde no se aplicó el T. harzianum, se manifestó la presencia del patógeno en los tallos (Ezziyyani et al., 2004; Sid el Ahmed et al., 1999).

Trichoderma viride es un organismo antagónico de hongos efectivo para el control de enfermedades causadas por patógenos de suelo, en cultivos de importancia económica. Este agente de biocontrol, cuando es aplicado con la semilla, coloniza la semilla y se multiplica sobre la superficie de la misma; no sólo mata los patógenos presentes sobre la superficie sino que también protege contra patógenos habitantes del suelo o en el sustrato del suelo para la vida del cultivo, con la acción de antibiosis y mico parasitismo (Chouaki et al., 2002).

El Metalaxil más Mancozeb es un producto comercial de dos tipos de fungicidas, uno de acción curativa y el otro protector contra Oomycetos. El metalaxyl actúa inhibiendo la síntesis de proteínas interfiriendo con la síntesis de ARN ribosomal y el mancozeb reacciona con los grupos sulfhídricos de los aminoácidos y las enzimas de las células fúngicas, resultando en la interrupción del metabolismo de los lípidos, de la respiración y de la producción de ATP (Grupo Silvestre, 2012).
El sulfato de cobre pentahidratado es un polímero orgánico natural que transporta al azufre y cobre a través del sistema vascular de las plantas, por lo que se considera de acción sistémica siendo efectiva para el control de la $P$. capsici. En el caso que las zoosporas de $P$. capsici empiecen su ataque cuando el sulfato de cobre pentahidratado ya se ha aplicado, el azufre y el cobre existente en el sistema inmunológico celular de las plantas impedirá la germinación de las zoosporas. Si la planta ha sido atacada con anterioridad a la aplicación del sulfato de cobre, al ingresar el azufre y el cobre al sistema inmunológico celular, destruirá las bacterias y hongos presentes en las células; es decir, el sulfato de cobre tendrá acción curativa (Laboratorio Ferrosalt, 2012). Niedman (2004), estudió la efectividad de diversos fungicidas en pimientos cultivados en macetas, bajo condiciones controladas, y encontró que el sulfato de cobre pentahidratado logró controlar en un $70 \%$ a $P$. capsici.

El fosfonato de potasio es recomendado para reducir el impacto de Phytophthora en diferentes cultivos; se puede aplicar en tratamiento del suelo, en pulverización foliar, en pulverización tronco o en inyección en el tronco a presión (Darvas et al., 1984; Guest et al., 1995).

El presente estudio tiene como objetivo evaluar la efectividad del uso de diversos biocontroladores y productos químicos comerciales en la prevención de las pudriciones de raíces del pimiento (Capsicum annuum) causado por Phytophthora capsici, tanto en condiciones de laboratorio como en condiciones de invernadero.

\section{Materiales y métodos Lugar del estudio}

Esta investigación se llevó a cabo en el laboratorio y en el Invernadero de Experimentación del Departamento Académico de Fitopatología de la Universidad Nacional Agraria La Molina, ubicada en el distrito de La Molina, Lima, Perú.

\section{Aislamiento e identificación del patógeno}

Se recolectaron plantas de ají páprika (Capsicum annuum) cv. Papri King con síntomas de pudriciones de cuello y raíces, procedentes de campos de cultivo de Ica. En el laboratorio se seccionaron porciones de tejido sano y enfermo de cuello, raíces y raicillas, los cuales fueron lavados con agua corriente, desinfestadas en hipoclorito de sodio al $0,5 \%$ por cinco minutos, se enjuagaron con agua destilada estéril y una vez secos fueron sembrados en placas petri que contenían el medio de cultivo Corn Meal Agar (CMA) al cual se le añadió pimaricina, ampicilina, rifampicina y benomilo (PAR-B) al momento del plaqueo. Las placas fueron incubadas a $25^{\circ} \mathrm{C}$ y se realizaron observaciones diarias. Las colonias obtenidas fueron transferidas a placas petri con medio de cultivo Agar Jugo V-8, incubadas a $25^{\circ} \mathrm{C}$ y cuando se logró un crecimiento micelial abundante, se cortaron rodajas del medio conteniendo el micelio y se colocaron 
en placas petri conteniendo solución de suelo al 1\% estéril para inducir la formación de los zoosporangios. Una vez obtenidos los zoosporangios y otras estructuras de reproducción, se realizaron las observaciones y mediciones a través del microscopio y luego, con ayuda de las claves de identificación de Erwin y Ribeiro (1996), se procedió a identificar a Phytophthora capsici.

\section{Prueba de patogénesis}

La prueba de patogenicidad se realizó en plantines de pimiento cv. Papri King que fueron proporcionados por el vivero Agrogénesis, ubicado en la ciudad de Chincha Alta. Los plantines fueron trasplantados en macetas de un kilogramo conteniendo una mezcla esterilizada de suelo agrícola, musgo y materia orgánica (compost) en proporción 1:1:1. Para la obtención del inóculo del $P$. capsici aislado, este fue repicado en medio de cultivo CMA y una vez obtenidas las colonias, se procedió a colocar rodajas del medio de cultivo con el pseudohongo en una solución de suelo estéril al $1 \%$ para inducir la formación de zoosporangios. Luego de haber obtenido los zoosporangios, se colocaron a temperatura de $5{ }^{\circ} \mathrm{C}$ por 30 minutos y luego a temperatura ambiente para inducir la formación y liberación de zoosporas. Una vez liberadas las zoosporas, se procedió a contabilizarlas con la cámara Neubauer y se estandarizó la suspensión a 10.000 zoosporas $/ \mathrm{ml}$.

La inoculación de las plantas de pimiento con $P$. capsici se realizó una semana después del trasplante en el invernadero. Cada plántula fue inoculada con $5 \mathrm{ml} \mathrm{de}$ suspensión de zoosporas de una concentración de $10^{4}$ zoosporas $\mathrm{ml}^{-1}$. Las plántulas fueron regadas en intervalos de tiempo que aseguraron el adecuado nivel de humedad del sustrato. Las plantas de pimiento inoculadas fueron evaluadas diariamente para observar la aparición de los síntomas secundarios de amarillamiento, marchitamiento y muerte ascendente, que indicaran posibles pudriciones de raíces. Las plantas con estos indicios fueron extraídas para observar los síntomas primarios de pudriciones del cuello y de las raíces. Las plantas con signos de pudrición de cuello y raíces fueron llevadas al Laboratorio de Fitopatología para proceder con aislarlas nuevamente del patógeno inoculado, de igual forma como se procedió en el proceso de aislamiento inicial.

\section{Prueba de efectividad en laboratorio de los productos químicos y biocontroladores ante $\boldsymbol{P}$. capsici.}

Se consideraron siete tratamientos los cuales se observan en la Tabla 1. Se realizaron pruebas de envenenamiento con los inductores de resistencia y fungicidas químicos y pruebas de enfrentamiento con los biocontroladores.

\section{Prueba de envenenamiento}

Los productos químicos utilizados fueron los indicados como tratamientos T1, T2 y T3 en la Tabla 1. Se procedió a pesar cada producto según la dosis comercial recomendada por los fabricantes y se añadió a cada producto $100 \mathrm{ml}$ de medio Papa Dextrosa Agar (PDA) líquido a punto de plaqueo; se homogeneizó y se procedió distribuirlo en placas petri. Una vez que el medio se solidificó y enfrió, se sembró al centro de cada placa una rodaja de PDA de $5 \mathrm{~mm}$ conteniendo micelio de $P$. capsici. El testigo de comparación fue sembrado de la misma forma pero en placas petri con medio de cultivo PDA sin ningún producto añadido. Las placas sembradas fueron incubadas a $25^{\circ} \mathrm{C}$ y cada 48 horas se realizó la evaluación del crecimiento micelial en los diferentes tratamientos.

\section{Prueba de enfrentamiento}

El primer paso fue la reactivación de los microorganismos biocontroladores indicados en los tratamientos T4, T5 y T6. Se procedió a pesar $1 \mathrm{~g}$ de cada producto y a cada uno de ellos se le adicionó $100 \mathrm{ml}$ de agua destilada estéril, se homogeneizó y se dejó reposar durante tres horas. Transcurrido el tiempo, se realizó la siembra respectiva de cada suspensión de biocontrolador en placas petri conteniendo PDA. Las placas sembradas fueron incubadas a $25^{\circ} \mathrm{C}$ por tres días.

De una colonia de $P$. capsici en PDA se extrajeron rodajas de $5 \mathrm{~mm}$ de diámetro y fueron colocados a un extremo de placas petri con medio PDA. Al otro extremo se sembró una rodaja de $5 \mathrm{~mm}$ conteniendo crecimiento del biocontrolador. Los testigos de comparación consistieron también en siembras con rodajas de PDA con los microorganismos colocados individualmente a un extremo de cada placa. Las placas sembradas fueron incubadas a $25^{\circ} \mathrm{C}$ y cada 48 horas se realizó la evaluación del crecimiento en los diferentes tratamientos. Para la prueba de efectividad en laboratorio se utilizó un

Tabla 1. Nombre de productos comerciales y dosis utilizadas en las pruebas in vitro pruebas in vitro

\begin{tabular}{|c|l|l|c|}
\hline Tratamientos & \multicolumn{1}{|c|}{ Nombre comercial } & \multicolumn{1}{|c|}{ Ingrediente activo } & Dosis empleada \\
\hline T1 & Ridomil Gold & Mancozeb $64 \%+$ Metalaxyl 4 \% & $7,5 \mathrm{~g} / \mathrm{L} \mathrm{de} \mathrm{agua}$ \\
\hline T2 & Fitopron & Fosfonato de potasio & $5 \mathrm{ml} / \mathrm{L}$ de agua. \\
\hline T3 & Sulcopenta & Sulfato de cobre pentahidratado & $2,5 \mathrm{ml} / \mathrm{L}$ de agua. \\
\hline T4 & Qs-Nd & Trichoderma viride & $2,5 \mathrm{~g} / \mathrm{L} \mathrm{de} \mathrm{agua.}$ \\
\hline T5 & Trichosil & Trichoderma harzianum & $1,5 \mathrm{~g} / \mathrm{L}$ de agua. \\
\hline T6 & Bio-Splent & Bacillus subtilis & $2,5 \mathrm{~g} / \mathrm{L} \mathrm{de} \mathrm{agua}$ \\
\hline T7 & TESTIGO & & ---- \\
\hline
\end{tabular}


Tabla 2. Tratamientos de productos contra $P$. capsici en invernadero

\begin{tabular}{|c|l|c|c|}
\hline Tratamiento & \multicolumn{1}{|c|}{ Productos comerciales } & Dosis de aplicación & Vías de aplicación \\
\hline T1 & Metalaxyl + Mancozeb & $7,5 \mathrm{~g} / \mathrm{L}$ de agua & Suelo \\
\hline T2 & Fosfonato de potasio & $5 \mathrm{ml} / \mathrm{L}$ de agua. & Foliar \\
\hline T3 & Sulfato de cobre pentahidratado & $2,5 \mathrm{ml} / \mathrm{L}$ de agua. & Suelo \\
\hline T4 & Trichoderma viride & $2,5 \mathrm{~g} / \mathrm{L}$ de agua. & Suelo \\
\hline T5 & Trichoderma harzianum & $1,5 \mathrm{~g} / \mathrm{L}$ de agua. & Suelo \\
\hline T6 & Bacillus subtilis & $2,5 \mathrm{~g} / \mathrm{L}$ de agua & Suelo \\
\hline T7 & Testigo: sustrato inoculado & Sin producto & ----- \\
\hline T8 & Testigo: sustrato sin inocular & Sin producto & ----- \\
\hline
\end{tabular}

Tabla 3. Escala de evaluación de severidad en raíces afectadas por $P$. capsici

\begin{tabular}{|c|l|c|}
\hline $\begin{array}{c}\text { Grado de } \\
\text { severidad }\end{array}$ & \multicolumn{1}{|c|}{ Descripción } & $\begin{array}{c}\text { Porcentaje de } \\
\text { severidad }\end{array}$ \\
\hline 0 & Raíz sana, planta vigorosa sana. & 0 \\
\hline 1 & $\begin{array}{l}\text { Color marrón oscuro que empieza a aparecer en el tallo o lesiones } \\
\text { marrones en las hojas. }\end{array}$ & $0-30$ \\
\hline 2 & Raíz oscura, con lesiones ligeramente muy pequeñas en el tallo. & $>30-50$ \\
\hline 3 & Raíz oscura, lesiones grandes en el tallo. & $>50-70$ \\
\hline 4 & Toda la planta marchita, crecimiento detenido. & $>70-90$ \\
\hline 5 & Planta muerta. & $>90-100$ \\
\hline
\end{tabular}

Fuente: Kim y Hwang (1992).

Diseño Completamente al Azar (DCA) considerando ocho tratamientos con cuatro repeticiones para cada uno (placa petri).

\section{Prueba de efectividad en invernadero de los productos} químicos y biocontroladores ante $\boldsymbol{P}$. capsici

Se utilizaron plantas de pimiento trasplantadas en macetas de un $1 \mathrm{~kg}$ del sustrato estéril compuesto de suelo agrícola, musgo y compost en proporción 1:1:1. Cada plántula fue inoculada con $5 \mathrm{ml}$ de suspensión de $10^{4}$ zoosporas $\mathrm{ml}^{-1} \mathrm{del}$ patógeno. En la Tabla 2 se observan los tratamientos empleados en condiciones de invernadero, asimismo como las dosis empleadas de cada uno, según la recomendación del fabricante, y la forma de aplicación. Los tratamientos testigos se hicieron considerando aquellos que tenían sustrato inoculado con $P$. capsici y sustrato $\sin$ inocular. En ambos no se realizaron aplicaciones de productos químicos ni biológicos.

Los biocontroladores se aplicaron 48 horas antes de la inoculación de $P$. capsici, y después cada ocho días totalizando tres aplicaciones; mientras que los fungicidas e inductores de resistencia (fertilizantes) se aplicaron 48 horas después de la inoculación de $P$. capsici y en tres momentos con un intervalo de 10 días entre cada aplicación.

Las variables que se evaluaron en la prueba de invernadero fueron: altura de planta, peso fresco de raíces, peso seco de raíces, incidencia de la enfermedad y la severidad de la enfermedad según la escala de evaluación para $P$. capsici de Kim \& Hwang (1992) (ver Tabla 3).

Para condiciones de invernadero, se utilizó el Diseño de Bloques Completamente al Azar (DBCA) considerando tres bloques con ocho tratamientos y cinco unidades experimentales (macetas) por cada tratamiento.

\section{Resultados y discusión}

Aislamiento e identificación del patógeno.

Se aisló e identificó a Phytophthora capsici L. según la Clave de Erwin y Ribeiro (1996) de las plantas de pimiento con síntomas de pudriciones de cuello y raíces, que fueron colectadas de Ica.

\section{Prueba de patogénesis}

El pseudohongo $P$. capsici inoculado en plántulas de pimiento produjo síntomas secundarios de marchitamiento, amarilleamiento y muerte ascendente; así como síntomas primarios de pudrición del cuello y pudrición de raíces. De las plantas con dichos síntomas se aisló nuevamente el $P$. capsici inoculado, corroborando que es el causante de las pudriciones de cuello y raíces en las plantas de pimiento recolectadas de Ica.

\section{Prueba de efectividad in vitro}

\section{Pruebas de envenenamiento}


Tabla 4. Crecimiento radial $(\mathrm{cm})$ de las colonias de $P$. capsici en las pruebas de envenenamiento con los productos químicos y prueba de comparación de Duncan al noveno día de crecimiento

\begin{tabular}{cccccccccc}
\hline & \multicolumn{1}{c}{ DÍAS DESPUÉS DE LA SIEMBRA } & & Duncan \\
TRATAMIENTOS & $\mathbf{2}$ & $\mathbf{3}$ & $\mathbf{4}$ & $\mathbf{5}$ & $\mathbf{6}$ & $\mathbf{7}$ & $\mathbf{8}$ & $\mathbf{9}$ & $(\mathbf{p}<\mathbf{0 , 0 5})$ \\
\hline Metalaxil + Mancozeb & 0,00 & 0,00 & 0,00 & 0,00 & 0,00 & 0,00 & 0,00 & 0,00 & $\mathrm{~d}$ \\
Fosfonato de Potasio & 0,00 & 0,10 & 0,10 & 0,10 & 0,10 & 0,10 & 0,10 & 0,10 & $\mathrm{c}$ \\
Sulfato de Cobre & 0,69 & 0,83 & 0,94 & 0,97 & 0,99 & 1,06 & 1,13 & 1,20 & $\mathrm{~b}$ \\
$\quad$ Testigo & 1,14 & 1,57 & 1,94 & 2,17 & 2,61 & 3,00 & 3,40 & 3,95 & $\mathrm{a}$ \\
\hline
\end{tabular}

Tabla 5. Crecimiento radial de las colonias de P. capsici y de los biocontroladores en las pruebas de enfrentamiento.

La Molina 2012

\begin{tabular}{|c|c|c|c|c|c|c|c|c|c|c|c|c|c|c|c|c|c|c|c|c|c|c|c|c|c|c|c|c|c|}
\hline \multirow[b]{3}{*}{ Tratamientos } & \multirow[b]{3}{*}{$\begin{array}{c}\text { Organismo } \\
\text { evaluado }\end{array}$} & \multicolumn{28}{|c|}{ Crecimiento radial $(\mathrm{cm})$} \\
\hline & & \multicolumn{28}{|c|}{ Días después de la siembra } \\
\hline & & \multicolumn{3}{|c|}{2} & \multicolumn{3}{|c|}{3} & \multicolumn{3}{|c|}{4} & \multicolumn{3}{|c|}{5} & \multicolumn{3}{|c|}{6} & \multicolumn{4}{|c|}{7} & \multicolumn{4}{|c|}{8} & \multicolumn{5}{|c|}{9} \\
\hline \multirow{4}{*}{$\begin{array}{l}\text { T. viride vs. } \\
\text { P. capsici }\end{array}$} & T. viride & 0,48 & $\mathrm{a}$ & & 1,10 & $\mathrm{a}$ & & 1,79 & $\mathrm{a}$ & & 2,45 & $\mathrm{a}$ & & 3,10 & $\mathrm{a}$ & & 3,53 & & $\mathrm{~b}$ & & 4,00 & & $\mathrm{~b}$ & & 4,23 & & $\mathrm{~b}$ & & \\
\hline & P. capsici & 0,40 & $\mathrm{a}$ & & 0,48 & & b & 0,76 & & $\mathrm{~b}$ & 0,95 & & $\mathrm{~b}$ & 1,13 & & $\mathrm{~b}$ & 1,19 & & & $\mathrm{c}$ & 1,25 & & & $\mathrm{c}$ & 1,23 & & & & d \\
\hline & $\begin{array}{l}\text { T. viride } \\
\text { (solo) }\end{array}$ & 0,48 & a & & 1,17 & $\mathrm{a}$ & & 1,85 & a & & 2,54 & $\mathrm{a}$ & & 3,23 & $\mathrm{a}$ & & 3,88 & $a$ & & & 4,40 & $a$ & & & 7,23 & $\mathrm{a}$ & & & \\
\hline & $\begin{array}{l}\text { P. capsici } \\
\text { (solo) }\end{array}$ & 0,30 & & $\mathrm{~b}$ & 0,51 & $\mathrm{~b}$ & & 0,71 & & $\mathrm{~b}$ & 0,92 & & $\mathrm{~b}$ & 1,13 & & b & 1,20 & & & c & 1,28 & & & c & 2,43 & & & c & \\
\hline \multirow{4}{*}{$\begin{array}{l}\text { T. harzianum } \\
\text { vs. } P \text {. capsici }\end{array}$} & $\begin{array}{c}T . \\
\text { harzianum }\end{array}$ & 0,20 & & $\mathrm{~b}$ & 1,10 & a & & 1,99 & a & & 2,89 & a & & 3,78 & $\mathrm{a}$ & & 4,20 & $a$ & & & 4,60 & $a$ & & & 4,63 & & $\mathrm{~b}$ & & \\
\hline & P. capsici & 0,35 & a & & 0,55 & & $\mathrm{~b}$ & 0,75 & & $\mathrm{~b}$ & 0,95 & & $\mathrm{~b}$ & 1,15 & & $\mathrm{~b}$ & 1,33 & & $\mathrm{~b}$ & & 1,53 & & $\mathrm{~b}$ & & 1,43 & & & & $\mathrm{~d}$ \\
\hline & $\begin{array}{c}T . \\
\text { harzianum } \\
\text { (solo) }\end{array}$ & 0,25 & a & $\mathrm{b}$ & 1,14 & $\mathrm{a}$ & & 2,01 & a & & 2,90 & a & & 3,78 & a & & 409 & $a$ & & & 4,40 & $a$ & & & 7,15 & a & & & \\
\hline & $\begin{array}{l}\text { P.capsici } \\
\text { (solo) }\end{array}$ & 030 & a & $\mathrm{b}$ & 0,51 & & $\mathrm{~b}$ & 0,71 & & $\mathrm{~b}$ & 0,92 & & $\mathrm{~b}$ & 1,13 & & $\mathrm{~b}$ & 1,20 & & $\mathrm{~b}$ & & 1,28 & & $\mathrm{~b}$ & & 2,43 & & & c & \\
\hline \multirow{4}{*}{$\begin{array}{l}\text { B. subtilis } \\
\text { vs. P. capsici }\end{array}$} & B. subtilis & 0,43 & a & & 0,84 & $\mathrm{a}$ & & 1,24 & a & $\mathrm{b}$ & 1,65 & a & $\mathrm{b}$ & 2,05 & a & $\mathrm{b}$ & 2,09 & $\mathrm{a}$ & $\mathrm{b}$ & & 2,13 & $\mathrm{a}$ & $\mathrm{b}$ & & 2,35 & a & & & \\
\hline & P. capsici & 0,30 & a & & 0,50 & $\mathrm{a}$ & & 0,70 & & $\mathrm{~b}$ & 0,90 & & $\mathrm{~b}$ & 1,10 & & $\mathrm{~b}$ & 1,23 & & $\mathrm{~b}$ & & 1,35 & & $\mathrm{~b}$ & & 1,93 & & $\mathrm{~b}$ & & \\
\hline & $\begin{array}{l}\text { B. subtilis } \\
\text { (solo) }\end{array}$ & 0,45 & $\mathrm{a}$ & & 0,98 & a & & 1,50 & a & & 2,03 & a & & 2,55 & a & & 2,55 & $a$ & & & 2,55 & $a$ & & & 2,55 & a & & & \\
\hline & $\begin{array}{l}\text { P. capsici } \\
\text { (solo) }\end{array}$ & 0,30 & $\mathrm{a}$ & & 0,51 & a & & 0,71 & & $\mathrm{~b}$ & 0,92 & & b & 1,13 & $\mathrm{~b}$ & & 1,20 & & $\mathrm{~b}$ & & 1,28 & & $\mathrm{~b}$ & & 2,43 & $\mathrm{a}$ & & & \\
\hline
\end{tabular}

En la Tabla 4 se puede observar los promedios de crecimiento radial de las colonias de $P$. capsici en diferentes días después de la siembra, con los distintos tratamientos con la respectiva Prueba de Duncan. En el tratamiento con Metalaxil $4 \%+$ Mancozeb $64 \%$ no hubo crecimiento de colonias desde el principio hasta el final de la evaluación. En el tratamiento con Fosfonato de Potasio el crecimiento radial comenzó a manifestarse el tercer día después de la siembra, manteniendo el promedio del mismo tamaño desde los cuatro a los nueve días. En el tratamiento con sulfato de cobre el crecimiento radial fue incrementándose conforme pasaron los días después de la siembra. En el testigo sin producto químico se observó el mayor crecimiento. La prueba de comparación de Duncan indica que los cuatro tratamientos químicos fueron estadísticamente diferentes a un nivel de significación del $5 \%$.

ElMetalaxil+Mancozeb resultó ser el tratamiento químico que tuvo mejor efecto inhibitorio en el crecimiento de $P$. capsici, haciéndolo al $100 \%$. Esto coincide también con los resultados obtenidos por Niedman (2004) quien evaluó el efecto de diferentes fungicidas en 14 aislamientos de
P. capsici encontrando que el Metalaxil logró inhibir el crecimiento de los aislamientos en un $95 \%$.

\section{Pruebas de enfrentamiento}

En la Tabla 5 se observan los promedios de las pruebas de enfrentamiento de $P$. capsici con los biocontroladores, desde los dos a los nueve d.d.s. se presentan los resultados de la Prueba de Comparación de Duncan a una probabilidad del $5 \%$.

En el enfrentamiento entre B. subtilis y $P$. capsici se observa que el tamaño de la colonia de $B$. subtilis frente a $P$. capsici fue similar al de su testigo $(2,35$ y $2,55 \mathrm{~cm}$ respectivamente) y estadísticamente iguales. La colonia de $P$. capsici tuvo un radio menor a $4,3 \mathrm{~mm}$ frente a $B$. subtilis.

La prueba de Duncan al $5 \%$ demostró que sí existen diferencias significativas entre los crecimientos de las colonias de $P$. capsici solas y con los biocontroladores. En todos los casos, los biocontroladores ejercieron un efecto negativo haciendo que las colonias sean de menor tamaño. Esto es debido, principalmente, a que los biocontroladores actúan en competición directa con 
Tabla 6. Promedios de incidencia de síntomas en Capsicum annuum después de ser inoculadas con $P$. capsici

\begin{tabular}{|c|c|c|c|c|c|c|c|c|c|c|c|c|c|c|c|c|c|c|c|}
\hline \multirow{2}{*}{\multicolumn{2}{|c|}{ Tratamientos }} & \multicolumn{18}{|c|}{ Días después de la inoculación (d.d.i) } \\
\hline & & \multicolumn{3}{|c|}{2} & \multicolumn{3}{|c|}{5} & \multicolumn{4}{|c|}{8} & \multicolumn{4}{|c|}{11} & \multicolumn{4}{|c|}{14} \\
\hline $\mathrm{T} 1$ & $\begin{array}{c}\text { Metalaxyl } \\
+ \\
\text { Mancozeb }\end{array}$ & 0,00 & & $\mathrm{~b}$ & 000 & & $\mathrm{~b}$ & 0,00 & & & $\mathrm{c}$ & 0,00 & & & $\mathrm{c}$ & 0,00 & & & $\mathrm{c}$ \\
\hline $\mathrm{T} 2$ & $\begin{array}{l}\text { Fosfonato } \\
\text { de potasio }\end{array}$ & 6,67 & $\mathrm{a}$ & $\mathrm{b}$ & 13,33 & $\mathrm{a}$ & $\mathrm{b}$ & 53,33 & $\mathrm{a}$ & $\mathrm{b}$ & & 73,33 & $\mathrm{a}$ & & & 86,67 & $\mathrm{a}$ & & \\
\hline $\mathrm{T} 3$ & $\begin{array}{l}\text { Sulfato de } \\
\text { cobre }\end{array}$ & 6,67 & $\mathrm{a}$ & $\mathrm{b}$ & 6,67 & $\mathrm{a}$ & $\mathrm{b}$ & 46,67 & & $\mathrm{~b}$ & & 53,33 & & $\mathrm{~b}$ & & 80,00 & & $\mathrm{~b}$ & \\
\hline $\mathrm{T} 4$ & T. viride & 26,67 & $\mathrm{a}$ & & 40,00 & $\mathrm{a}$ & & 60,00 & $\mathrm{a}$ & & & 60,00 & $\mathrm{a}$ & $\mathrm{b}$ & & 86,67 & $\mathrm{a}$ & & \\
\hline $\mathrm{T} 5$ & $\begin{array}{c}T . \\
\text { harzianum }\end{array}$ & 20,00 & $\mathrm{a}$ & $\mathrm{b}$ & 20,00 & $\mathrm{a}$ & $\mathrm{b}$ & 66,67 & $\mathrm{a}$ & & & 73,33 & $\mathrm{a}$ & & & 100,00 & $\mathrm{a}$ & & \\
\hline $\mathrm{T} 6$ & B. subtilis & 26,67 & $\mathrm{a}$ & & 33,33 & $\mathrm{a}$ & $\mathrm{b}$ & 53,33 & $\mathrm{a}$ & $\mathrm{b}$ & & 60,00 & $\mathrm{a}$ & $\mathrm{b}$ & & 86,67 & $\mathrm{a}$ & & \\
\hline $\mathrm{T} 7$ & $\begin{array}{c}\text { Testigo } \\
\text { inoculado }\end{array}$ & 13,33 & $\mathrm{a}$ & $\mathrm{b}$ & 13,33 & $\mathrm{a}$ & $\mathrm{b}$ & 66,67 & $\mathrm{a}$ & & & 66,67 & $\mathrm{a}$ & & & 100,00 & $\mathrm{a}$ & & \\
\hline $\mathrm{T} 8$ & $\begin{array}{l}\text { Testigo } \\
\text { absoluto }\end{array}$ & 0,00 & & $\mathrm{~b}$ & 0,00 & & $\mathrm{~b}$ & 0,00 & & & $\mathrm{c}$ & 0,00 & & & $\mathrm{c}$ & 0,00 & & & $\mathrm{c}$ \\
\hline
\end{tabular}

el patógeno por los nutrientes y por el espacio donde habitan; asimismo, por la producción de antibióticos que evitan el crecimiento de otros organismos alrededor. En el caso de los Trichoderma spp., se observó también un efecto de parasitismo ya que son micoparásitos y agresivos competidores de patógenos (Sid, Pérez, Egea y Candela, 1999; Sid, Ezziyyani, Pérez y Candela, 2003).

\section{Prueba de efectividad en invernadero}

\section{Incidencia.}

En la Tabla 6 se muestran los resultados del porcentaje de incidencia encontradas en las plántulas de pimiento cv. Papri King a los 2, 5, 8, 11 y 14 días después de la inoculación (d.d.i) observándose diferencias estadísticamente significativas entre los tratamientos. El tratamiento con Metalaxyl + Mancozeb tuvo un comportamiento idéntico al testigo sin inoculación de $P$. capsici, presentando un $0 \%$ de incidencia. Mientras que a los cinco días después de la inoculación, la acción de $P$. capsici aumentó considerablemente en el tratamiento con

Tabla 7. Promedios de severidad en las pruebas de efectividad en plántulas de Capsicum annuum después de 14 días de inoculación y Prueba de Duncan

\begin{tabular}{|l|c|c|c|c|}
\hline \multicolumn{1}{|c|}{ TRATAMIENTOS } & \multicolumn{5}{c|}{$\begin{array}{c}\text { SEVERIDAD } \\
\text { (grados) }\end{array}$} \\
\hline Metalaxyl 4\% + Mancozeb 64\% & 0 & & & c \\
\hline Fosfonato de potasio & 5 & a & & \\
\hline Sulfato de cobre & 4 & & b & \\
\hline T. viride & 5 & a & & \\
\hline T. harzianum & 5 & a & & \\
\hline B. subtilis & 5 & a & & \\
\hline Testigo inoculado & 5 & a & & \\
\hline Testigo absoluto & 0 & & & c \\
\hline
\end{tabular}

T. viride, hasta llegar a un $40 \%$ de incidencia, seguida de B. subtillis (33,33\%), T. harzianum (20\%), fosfonato de potasio $(13,33 \%)$, el testigo inoculado $(13,33 \%)$ y el sulfato de cobre $(6,67 \%)$. Entre los 8 y los 11 d.d.i, el efecto del patógeno fue manifiesto; sin embargo, el porcentaje de incidencia se mantuvo igual con $T$. viride y el testigo inoculado. Se puede apreciar que a los 14 d.d.i, no hubo diferencias estadísticamente significativas $(\mathrm{P}>0,05)$ entre los tratamientos con fosfonato de potasio, T. viride, T. harzianum, B. subtilis y el testigo inoculado; no obstante, T. harzianum tuvo un comportamiento similar al testigo inoculado, presentando ambos $100 \%$ de incidencia.

\section{Severidad}

En la Tabla 7 se presentan los resultados para severidad representados mediante la escala de evaluación en grados del 0 a 5 según Tabla 3. La evaluación de severidad fue realizada a los 14 días después de la inoculación y se observó que existieron diferencias estadísticamente significativas $(\mathrm{P}<0,05)$ para los diferentes tratamientos. Sin embargo, no existieron diferencias estadísticamente significativas $(\mathrm{P}>0,05)$ entre los tratamientos con fosfonato de potasio, T. viride, T. harzianum, B. subtilis y el testigo inoculado, presentando todos estos tratamientos una severidad de grado 5, es decir todas las plántulas resultaron muertas. En el tratamiento con sulfato de cobre, se observa una severidad de grado 4 , es decir las plantas se marchitaron y su crecimiento se detuvo. Se puede apreciar también en las figuras 9 y 10 que las plántulas tratadas con Metalaxyl + Mancozeb no presentaron los síntomas de la enfermedad, por lo que la severidad tuvo grado cero que es el equivalente a una planta sana, igual que en el testigo absoluto que también tuvo una severidad de grado cero.

Tanto en la incidencia como en la severidad, ninguno de los productos químicos ni los biológicos superaron 
Tabla 8. Altura de planta $(\mathrm{cm})$ antes y después de la inoculación de $P$. capsici en plántulas de Capsicum en condiciones de invernadero

\begin{tabular}{|l|c|c|l|c|c|c|c|c|}
\hline \multirow{2}{*}{ TRATAMIENTOS } & \multicolumn{6}{|c|}{ Altura antes de la Inoculación } & \multicolumn{3}{c|}{ Altura después de la Inoculación } & (cm) & Crecimiento (cm) \\
\hline Metalaxyl + Mancozeb & 24,93 & a & b & 31,93 & a & & & 7,00 \\
\hline Fosfonato de potasio & 26,47 & a & & 29,27 & & b & c & 2,80 \\
\hline Sulfato de cobre & 25,53 & a & b & 28,60 & & b & c & 3,07 \\
\hline T. viride & 23,73 & a & b & 27,67 & & b & c & 3,94 \\
\hline T. harzianum & 24,80 & a & b & 28,33 & & b & c & 3,53 \\
\hline B. subtilis & 23,33 & & b & 27,20 & & & c & 3,87 \\
\hline Testigo inoculado & 24,67 & a & b & 28,40 & & b & c & 3,73 \\
\hline Testigo absoluto & 25,27 & a & b & 30,27 & & b & & 5,00 \\
\hline
\end{tabular}

Tabla 9. Promedio de pesos fresco y seco de las raíces de Capscicum annuum

\begin{tabular}{|l|l|l|l|l|l|l|l|l|}
\hline \multicolumn{1}{|c|}{ TRATAMIENTOS } & \multicolumn{3}{c|}{ PESO FRESCO (g) } & \multicolumn{3}{c|}{ PESO SECO (g) } \\
\hline Metalaxyl & 2,61 & $\mathrm{a}$ & & & 0,91 & $\mathrm{a}$ & & \\
Fosfonato de potasio & 0,49 & & $\mathrm{~b}$ & $\mathrm{c}$ & 0,23 & & & $\mathrm{c}$ \\
\hline Sulfato de cobre & 0,68 & & $\mathrm{~b}$ & & 0,26 & & & $\mathrm{c}$ \\
\hline T. viride & 0,47 & & $\mathrm{~b}$ & $\mathrm{c}$ & 0,18 & & & $\mathrm{c}$ \\
\hline T. harzianum & 0,36 & & & $\mathrm{c}$ & 0,17 & & & $\mathrm{c}$ \\
\hline B. subtilis & 0,48 & & $\mathrm{~b}$ & $\mathrm{c}$ & 0,19 & & & $\mathrm{c}$ \\
\hline Testigo inoculado & 0,32 & & & $\mathrm{c}$ & 017 & & & $\mathrm{c}$ \\
\hline Testigo absoluto & 2,17 & $\mathrm{a}$ & & & 0,61 & & $\mathrm{~b}$ & \\
\hline
\end{tabular}

al fungicida Metalaxyl + Mancozeb, que previno la aparición de la enfermedad en un 100 \%. Esto se debió a que ambos productos tienen acción directa sobre la fisiología de P. capsici, afectando la biosíntesis de los ácidos ribonucleicos del patógeno y por ende su multiplicación (La Torre, 2001).

\section{Altura de planta}

Las evaluaciones de la altura de planta se realizaron desde la base del cuello hasta la yema terminal de las plántulas, 10 días antes de la inoculación del patógeno y 10 días después de la última aplicación de los productos comerciales. En la Tabla 8 se presentan los resultados de las evaluaciones realizadas antes y después de la inoculación del patógeno. Al realizar las evaluaciones de altura en las plántulas inoculadas con el patógeno, se observan diferencias estadísticamente significativas entre los diferentes tratamientos. Se encontró que el tratamiento que tuvo la mayor altura fue el Metalaxyl + Mancozeb, seguido del testigo absoluto, fosfonato de potasio, el testigo inoculado, sulfato de cobre, $T$. harzianum, T. viride y B. subtilis, respectivamente. Se observa que el tratamiento con Metalaxyl + Mancozeb logró un crecimiento adicional de $7 \mathrm{~cm}$ en comparación con los otros tratamientos, incluso superando al testigo absoluto, que fue de $5 \mathrm{~cm}$ adicionales, mientras que el fosfonato de potasio experimentó el menor crecimiento adicional con $2,80 \mathrm{~cm}$.

\section{Peso fresco y peso seco de las raíces}

En la Tabla 9 se observan diferencias estadísticamente significativas para los pesos frescos de las raíces de Capsicum, encontrándose que el tratamiento con Metalaxyl + Mancozeb y el testigo absoluto obtuvieron los mayores pesos frescos de raíces seguidos por el sulfato de cobre, el fosfonato de potasio, B. subtilis, T. viride, $T$. harzianum y el testigo inoculado, respectivamente. En lo referente a los pesos secos de las raíces, el mejor tratamiento resultó el Metalaxyl + Mancozeb seguido del testigo absoluto, sulfato de cobre, sosfonato de potasio, B. subtilis, T. viride, T. harzianum y el testigo inoculado. No se encontraron diferencias significativas entre el fosfonato de potasio, el sulfato de cobre, el T. viride, el $T$. harzianum, el B. subtilis y el testigo inoculado.

Se aprecia que el fungicida Metalaxyl + Mancozeb fue el único producto químico con el cual se tuvo una planta con mayor altura, con mayor peso fresco y mayor peso seco de las raíces, debido principalmente a que este fungicida fue el único tratamiento que previno la aparición de las pudriciones de raíces por $P$. capsici en las plantas de pimiento; por lo tanto, todas las plantas que recibieron este tratamiento estuvieron sanas y pudieron crecer y desarrollar sin mayor interferencia patógena. Los inductores de resistencia como los controladores biológicos manifestaron una altura de planta, peso fresco y peso seco similar, deduciéndose que, debido al daño 
ocasionado por el patógeno, el crecimiento radicular se vio afectado manifestándose en una menor absorción de agua y de nutrientes y, por consiguiente, menor desarrollo general de las plantas. Los valores de incidencia, severidad, altura de planta, peso fresco y peso seco, para el tratamiento con Metalaxyl + Mancozeb, indican que fue el tratamiento más efectivo para el control de $P$. capsici en el cultivar Papri King en condiciones de invernadero.

\section{Conclusiones}

El fungicida formulado con Metalaxyl + Mancozeb inhibió el crecimiento total de Phytophthora capsici, resultando ser más efectivo contra este patógeno en condiciones de laboratorio; mientras que el fosfonato de potasio, el sulfato de cobre, los biocontroladores: T. viride, T. harzianum y B. subtilis no resultaron ser efectivos para inhibir el crecimiento de $P$. capsici, in vitro. En condiciones de invernadero el fungicida Metalaxyl + Mancozeb evitó o previno eficientemente la pudrición de las raíces causada por Phytophthora capsici en pimientos. El fosfonato de potasio, el sulfato de cobre, los biocontroladores: T. viride, T. harzianum y $B$. subtilis no fueron efectivos para prevenir la aparición de la pudrición de las raíces causada por $P$. capsici en pimientos.

\section{Literatura citada}

Agrios, G. 1998. Fitopatología. (2da. edición). México: Editorial Limusa.

Casas, A. 1994. Exportación de pimiento. En: Inflación y Devaluación. 125: 18-19.

Chouaki, V.; Lavarde, L.; Raccurt, C.P. y Hennequin, C. 2002. Clinical Infectious Diseases, 35: 1360-1367.

Darvas, J.M.; Toerien, J.C. y Milne, D.C. 1984. Control of avocado root rot by trunk injection with fosetyl-Al. Plant Disease, 68: 691-693.

Erwin, D. y Ribeiro, O. 1996. Phytophthora diseases worldwide. Minnesota, USA: APS press.

Ezziyyani, C.; Pérez, S.; Ahmed, S.; Requena, M. y Candela, M.E. 2004. Trichoderma harzianum como biofungicida para el biocontrol de Phytophthora capsici en plantas de pimiento (Capsicum annuum L.). Anales de biología, ISSN 26: 35-45.

Farr, D. y Bills, G.F. 1989. Fungi on plants and Plant Products in the United States. St. Paul, Minnesota, U.S.A.: APS.

Guest, D.I.; Pegg, K.G. y Whiley, A.W. 1995. Control of Phytophthora diseases of three crops using trunk-injected phosphonates. Horticulture, 17: 299-330.
Grupo Silvestre. 2012. Ficha técnica comercial. 63 pp.

Laboratorio Ferrosalt. 2012. Sulfato de cobre pentahidratado. Sulcopenta. Disponible en: http:// www.ferrosalt.com.pe/archivos/agricola/fungicidas/ sulcopenta.pdf.

Lara, J. 2006. Manual del Cultivo de Páprika. Instituto Peruano del Espárrago y Hortalizas - IPEH.

La Torre, B. 2001. Fungicidas y Nematicidas. Santiago, Chile: Pontifica Universidad Católica de Chile.

Mont, R.M. 1993. Principios del control de enfermedades de las plantas. Lima, Perú: Edit. Talleres del Centro Preuniversitario, Universidad Nacional Agraria La Molina.

Nicho, S.P. 2004. Cultivo de ají escabeche. Estación Experimental Donoso-Huaral. Huaral, Perú: Programa Nacional de Investigación en Hortalizas.

Niedman, L.M. 2004. Etimología de la enfermedad "Pudrición al cuello" en pimentón (Capsicum annuum var. Grossum) y efecto de cinco fungicidas sobre el control in vitro e in vivo del agente causal. (Tesis de grado). Universidad de Talca. Chile. Disponible en: http://dspace.utalca.cl/retrieve/1108/LNiedmannL.pdf .

Ocampo, M. 2003. Control biológico de Phytophthora capsici León en pimiento (Capsicum annuum L.). (Tesis de grado). Universidad Nacional Agraria La Molina. Lima, Perú.

Sid, A.A.; Ezziyyani, M.; Pérez, S.C. y Candela, M.E. 2003. Effect of chitin on biological control activity of Bacillus spp. and Trichoderma harzianum against root rot disease in pepper (Capsicum annuum) plants. European Journal of Plant Pathology, 109: 418-426.

Sid,A.A.; Pérez, S.C.; Egea, G.C.y Candela, C.E. 1999. Evaluation of the capacity of Trichoderma harzianum in controlling rot caused by Phytophthora capsici in pepper plants. Plant Pathology, 48: 58-65.

Stamps, J. 1985. Phytophthora capsici. Descriptions of Pathogenic Fungi and Bacteria. Commonwealth Mycological Institute (C.M.I).

Valiela, F. 1978. Introducción a la Fitopatología. Buenos Aires, Argentina: Colección científica del INTA. 